\title{
HERRAMIENTAS PARA DETECTAR LA SITUACIÓN IGUALITARIA EN LAS MUJERES Y HOMBRES EN LAS EMPRESAS
}

\author{
TOOLS TO DETECT EQUAL STATUS FOR WOMEN AND MEN IN BUSINESS
}

\author{
Margarita Artal Serrat ${ }^{1}$ \\ Carmen Cesteros Fernández ${ }^{2}$
}

\section{RESUMEN}

Las dificultades salariales, los aspectos socio-laborales son especialmente difíciles de plasmar y es por ello que, en este artículo las autoras plantean introducir en las empresas peruanas herramientas para desarrollar acciones de igualdad que consideran fundamentales y que está dando resultados satisfactorios en empresas de otros países, conformando un mejor clima laboral que redunda en una mejora de los resultados empresariales e incide en una mejora de las condiciones de trabajo desde la perspectiva de la equidad de género.

En el artículo también se dan referencias de como las leyes, las medidas sociales y empresariales son las que deben ayudar a que existan herramientas que consigan la igualdad de oportunidades entre mujeres y hombres en el mundo laboral. Mostrar los beneficios de políticas y acciones igualitarias que impida la fuga de talento, también es importante para percibir dichos beneficios. Se muestran medidas que ya están implementadas y que ayudaran a sensibilizar a las empresas peruana de la importancia de introducir dichas herramientas y poderlas evaluar para conseguir la igualdad de oportunidades en el seno de su cultura empresarial.

\section{Palabras clave}

Igualdad, empresas, talento, género, sensibilización

\section{ABSTRACT}

The wage difficulties, the socio-labour aspects are especially difficult to reflect and that is why, in this article the authors plan to introduce tools in Peruvian companies to develop actions of equality that they consider fundamental and that is giving satisfactory results in companies from other countries, creating a better work environment that results in an improvement of business results and impacts on the improvement of working conditions from the perspective of gender equity.

In the article there are references of how laws, social and business measures are the ones that should help tools to exist to achieve equality of opportunity between women and men in the working environment. Showing the benefits of egalitarian policies and actions to prevent the brain drain, it is also important to receive such benefits. It shows measures that are already implemented and that will help to make aware the Peruvian companies of the importance of introducing such tools and evaluate them to achieve equality of opportunity within its corporate culture.

\section{Keywords}

Equality, companies, talent, gender increasing awareness

1 Máster en Gestión y Política Universitaria, Licenciada en Pedagogía, Directora-Socia de ARTAU S.L., Directora-Socia de EMPRENUOVA S.L., Secretaria General de la Asociación Europea WiTEC - Women in Science, Engineering and Technology (SET). Directora del Programa Dona (Programa Mujer) en la Universitat Politécnica de Catalunya (UPC).

2 Gerente de The International Institute for Scientific Research. Consultora. Coach certificada. Liicenciada en Marketing y Finanzas. Diplomas en Recursos Humanos, Gestión de personal y Seguros Sociales. Presidenta de la Asociación Empresarial de Economía en Femenino. Vicepresidenta de la Cámara de comercio Hispano-Chilena. 


\section{INTRODUCCIÓN}

En el análisis de la evolución general del desarrollo democrático en los países latinoamericanos, es decir la dimensión que mide el desarrollo democrático desde la óptica del ciudadano o desde las condiciones para ejercer sus derechos y libertades, Perú junto a Argentina, Panamá y Bolivia completa el segundo grupo mejor posicionado que supera el promedio de la región de América Latina. El grupo mejor posicionado es el liderado por Chile, Costa Rica y Uruguay.

Según el IDD-LAT (Índice de desarrollo democrático de América Latina 2012) una de las fortalezas de estos países es la participación creciente de las mujeres en las decisiones políticas. La mayoría de paíseslatinoamericanos se enfrentan a grandes retos de crecimiento, productivos, económicos, sociales, laborales. La gran pregunta es cómo se cerrará la brecha que todavía está presente en muchos países de Latinoamérica. Son necesarias inevitables mejoras de la participación pública, para estimular la actividad, productiva, exportadora, económica... pero sobre todo se requiere entender que un modelo basado en la inequidad (de todo tipo, también la de las mujeres y los hombres) va a ser un modelo que no sobrevivirá por mucho tiempo.

Con el fuerte proceso expansivo que están viviendo los países latinoamericanos se crea un periodo muy importante para avanzar en las políticas de igualdad de oportunidades y aunque existen diferencias significativas entre unos países y otros, sí se está produciendo un proceso de sensibilización de la sociedad hacia el tema.

Por el contrario en Europa se está en un proceso contrario de recesión económica que está afectando a la igualdad entre hombres y mujeres, produciéndose un retroceso en algunos aspectos, especialmente en aquellos que no se había consolidado.

Por eso, a pesar de las legislaciones existentes, conviene en los periodos de bonanza asentar lo conseguido. En uno de los aspectos que queremos incidir es en las políticas de igualdad dentro de las empresas y del mundo económico, donde los avances son más lentos y se asientan sobre bases más precarias. Las diferencias salariales, los aspectos sociolaborales son especialmente difíciles de plasmar y es por ello que, nos proponemos plantear una herramienta que consideramos fundamental y que está dando resultados satisfactorios dentro de las empresas conformando un mejor clima laboral que redunda en una mejora de los resultados empresariales $e$ incide en una mejora de las condiciones de trabajo de hombres y mujeres.

Esta herramienta es lo que se conoce como planes de igualdad de oportunidades en las empresas. Pero, ¿qué es un plan de igualdad en las empresas? Los planes de igualdad de las empresas son un conjunto ordenado de medidas, adoptadas después de realizar un diagnóstico de situación, tendentes a alcanzar en la empresa la igualdad de trato y de oportunidades entre mujeres y hombres y a eliminar la discriminación por razón de sexo. Es una herramienta estratégica para la realización del potencial de las personas de una empresa (y por tanto el no desprecio de una parte del talento existente) que será una cuestión que se reflejará en la cuenta de resultados

Se lleva a cabo mediante un proceso metodológico de diagnóstico que sirve para determinar, desde la perspectiva de género, planes de acción de la empresa para integrar la igualdad de oportunidades en la organización y realizar un seguimiento mediante indicadores de género del desarrollo de las medidas implantadas. Tiene que estar implicada toda la organización y es fundamental la participación y el apoyo expreso de la dirección.

Con respecto a la situación de la mujer en el Perú Los datos recogidos en el año 2011 por UNIQUE nos indican que la mujer peruana representa el 45,6 \% de la población económicamente activa - PEA (7 millones 48 mil mujeres). La PEA femenina en el área urbana es de $46 \%$ y $44,9 \%$ en el área rural.

La tasa de actividad económica es para los hombres $82,3 \%$ y para las mujeres $64,8 \%$. La 
tasa de actividad de la mujer en el área urbana alcanza el 63,0\% y de la mujer del área rural, $4.1 \% .^{3}$

El 71,4 \% de la PEA ocupada femenina urbana se concentra en empresas que tienen entre 1 a 10 trabajadores, el $21,1 \%$ en establecimientos de 51 y más trabajadores y el 7,5\% en empresas de 11 a 50 trabajadores ${ }^{4}$.

El $67 \%$ de las mujeres frente al $53 \%$ de los hombres trabajan en el sector informal. Por cada 100 hombres hay 108 mujeres en pobreza extrema. ${ }^{5}$

El ingreso promedio de la PEA femenina (S/. 826.8) en el 2010, representó el $65,0 \%{ }^{6} \mathrm{del}$ ingreso promedio de la PEA masculina (S/. 1 $323,3)$.

El 45\% de las mujeres están trabajando, pero el $14 \%$ lo hace exclusivamente a tiempo completo y casi el mismo porcentaje, el $13 \%$, trabaja a media jornada.

El $46 \%$ de la población femenina trabaja solo en las tareas domesticas del hogar.

En el año 2010, el tiempo que dedican las mujeres a las actividades domésticas no remuneradas es en promedio semanal, el doble que el tiempo que dedican los hombres (39 horas con 28 minutos las mujeres y 15 horas con 53 minutos, los hombres) ${ }^{7}$.

La distribución de la carga global de trabajo6 es desigual entre mujeres y hombres. Las mujeres dedican casi la mitad de su tiempo de trabajo, al trabajo no remunerado (52\%), mientras que los hombres solo dedican $24 \%$ de su tiempo, esto explica por qué los hombres tienen mayor oportunidad que las mujeres para obtener ingresos ${ }^{8}$.

La insuficiencia de servicios públicos para el cuidado de personas, como guarderías y centros de atención para adultos mayores y personas totalmente dependientes, incrementa el tiempo de trabajo no remunerado de las mujeres. Es así que, el tiempo dedicado al cuidado de niños, niñas y adolescentes que destinan las mujeres afecta su ciclo laboral, a diferencia de los hombres, que no asumen las responsabilidades del cuidado de manera equitativa9.

Las mujeres, a pesar de conformar el $45 \%$ de la PEA activa se encuentran en puestos de trabajo que no cuentan con protección legal laboral, ya que se ubican en regímenes laborales especiales como trabajadoras del hogar, trabajadoras familiares no remuneradas, trabajadoras asalariadas de la agroexportación, conserveras, entre otras. Por ello, las mujeres que trabajan cuentan con reducidas posibilidades de participación en procesos de negociación colectiva y en organizaciones sindicales, derechos laborales respaldados por la OIT.

Las mujeres del área rural, dedican 19 horas con 41 minutos promedio semanal a cocinar, y si consideramos que el 68,5\% de los hogares rurales cocinan con leña y bosta y el $16 \%$ lo hace con estiércol ${ }^{10}$, podemos entender el importante papel que tendrían las mujeres en la preservación del medio ambiente y en la salud pública, en la ejecución de políticas de cambio a tecnologías más limpias y menos contaminantes.

\footnotetext{
1 INEI. Estadísticas con enfoque de género. Lima 2011. www.inei.gob.pe/web/Biblioinei/Boletines

Ibid. p. 58

Fuente: CEPAL, 2008: Estadísticas de América Latina y El Caribe.

Ibid. p. 68

MIMDES-INEI. I Encuesta Nacional de Uso del Tiempo. 2010

8 Suma del trabajo remunerado y no remunerado (es trabajo tanto el que se realiza en el mercado laboral a cambio de una remuneración, como el que se realiza en el ámbito doméstico para garantizar el bienestar y la vida). MIMDES. Brechas de género en el uso del tiempo. Freyre, M y López, E. 2010.

9 MIMDES-INEI. I Encuesta Nacional de Uso del Tiempo. 2010.

10 MIMDES. Brechas de Género en el Uso del Tiempo. Freyre, M. López, E. Lima 2011. Y MIMDES-INEI. Encuesta Uso del Tiempo. 2010.
} 
Según la información de COFOPRI ${ }^{11}$, el 2011 se entregaron 68,881 títulos de propiedad a mujeres. El 20.3\% de unidades agropecuarias son dirigidas por mujeres, pero sólo el $4.7 \%$ de las productoras rurales tiene su título de propiedad $^{12}$.

El 10\% de mujeres se declara empresaria.
De todos estos porcentajes el que llama más la atención es el $5 \%$ de mujeres estudiantes, sobre todo si se contrasta con $39 \%$ de mujeres que declaran que les gustaría estudiar una carrera.

Finalmente otro dato a tener en cuenta nos recuerda que el $76 \%$ de las mujeres desearía trabajar pero a media jornada

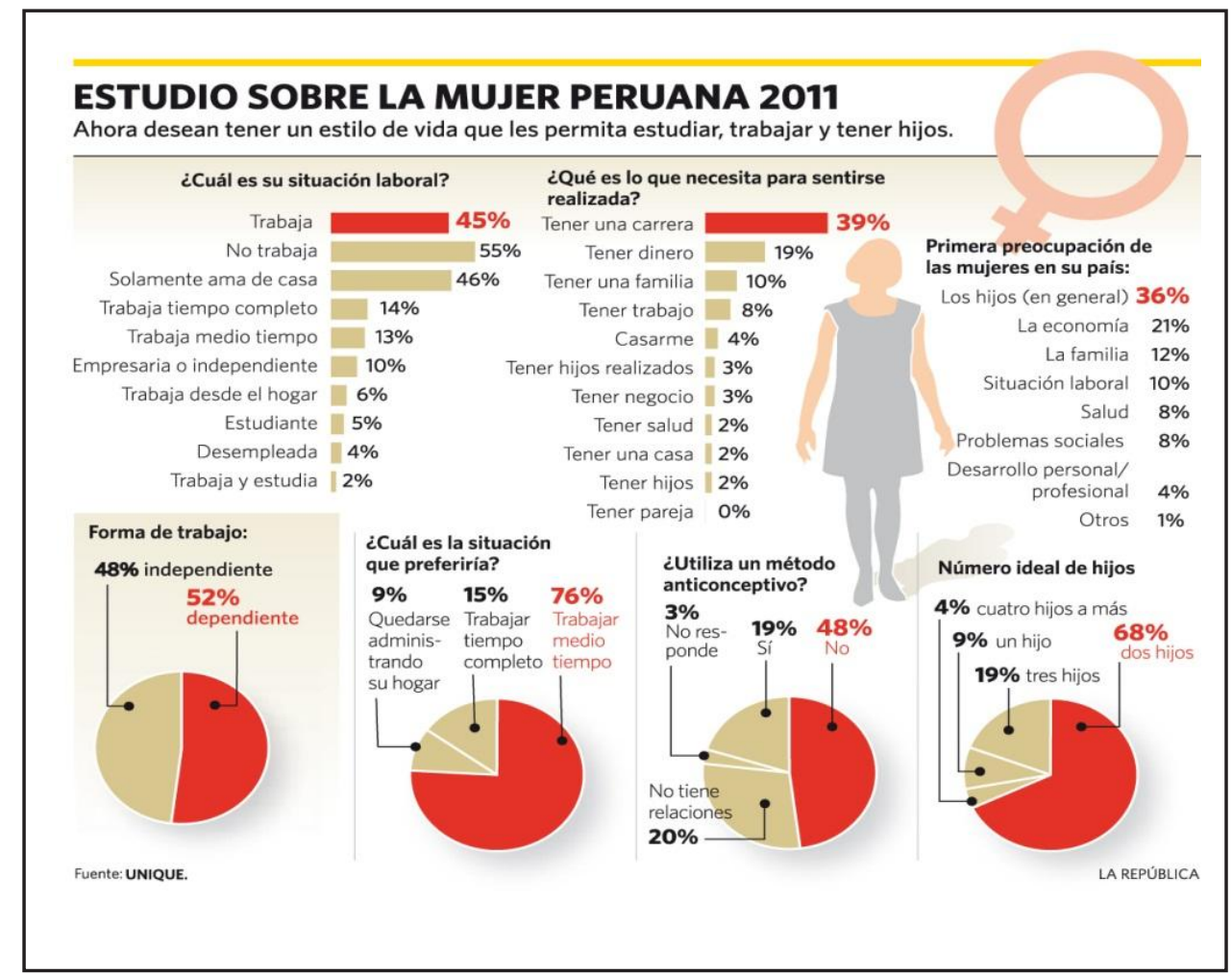

\section{LA INTRODUCCIÓN DE DATOS ¿PARA QUÉ?}

En primer lugar que Perú es un país que se encuentra en un importante momento de crecimiento económico $y$, a su vez con un importante desarrollo de libertades políticas y sociales. Este tipo de crecimiento siempre ayuda a pensar en una sociedad lo más igualitaria posible desde la perspectiva de la igualdad social, pero también desde la perspectiva entre mujeres y hombres.

Segundo aspecto importante, cuando una sociedad avanza como lo está haciendo la peruana en sus parámetros económicos y laborales, no puede dejar de lado a la mujer ni a su necesaria incorporación en el mundo laboral y para ello es imprescindible que esta incorporación, que llegará a ser masiva y sin posibilidad de dar marcha atrás, sea preparada. No se puede incurrir en los mismos errores de algunos países europeos cuando las mujeres fueron incorporadas de forma masiva al mercado laboral, pero sin que se acompañaran las suficientes políticas de igualdad y los mecanismos legales efectivos contra la discriminación, en algunos países eso significó entre otras cosas, un descenso del nivel salarial

\footnotetext{
11 El Proyecto Especial de Titulación de Tierras y Catastro Rural - PETT fue incorporado al Organismo de Formalización de la Propiedad Informal - COFOPRI, mediante un proceso de fusión por absorción aprobado por Decreto Supremo $\mathrm{N}^{\circ} 005$ 2007-VIVIENDA.

12 Ministerio de Vivienda, Construcción y Saneamiento. Informe DS 027-2007-PCM. Enero 2012.
} 
medio $^{13}$, (Vicenç Navarro) pero también la incorporación femenina poco cualificada, y escasamente valorada a nivel laboral social y claro económico también.

El tercer aspecto que hay que destacar es que existe en estos momentos en Perú un importante movimiento de grupos de mujeres que ya están trabajando para posicionarse y alertar a la sociedad en general de la necesidad de visibilizar y mejorar la situación de la mujer en muchos ámbitos. Pero este trabajo debe filtrarse al mundo laboral, a las empresas que deben contratar mujeres y organizar las estructuras laborales para que las mujeres reúnan las condiciones necesarias en su entorno para poder llevar a cabo las jornadas de trabajo con toda normalidad.

Este artículo se va a centrar en analizar qué se puede hacer para que las mujeres se incorporen en los lugares de trabajo y las empresas sean "acogedoras" no solamente para ellas, sino también para sus compañeros hombres. Esto se agrava, como ya hemos planteado en los datos anteriormente expuestos, por la insuficiencia de servicios públicos para el cuidado de personas, como guarderías y centros de atención para adultos mayores y personas totalmente dependientes lo que incrementa el tiempo de trabajo no remunerado de las mujeres afectando a su ámbito laboral, a diferencia de los hombres, que no asumen las responsabilidades del cuidado de manera equitativa

\section{¿CUÁL ES EL MARCO NORMATIVO EN PERÚ?}

La institucionalización de políticas públicas de igualdad de género en Perú se sustenta en una serie de normas nacionales e internacionales; así como en compromisos políticos asumidos por el Estado peruano en foros nacionales $e$ internacionales.

La Constitución Política del Perú vigente (1993) reconoce el derecho a la igualdad y no discriminación por razón de sexo. Este texto fue reformado parcialmente en el año 2002 y en la reforma del artículo 191 se introdujo el principio de representación por género. El actual marco normativo sobre la igualdad de oportunidades entre hombres y mujeres, en Perú, está enmarcado en la Ley de Igualdad de oportunidades que fue aprobada el 16 de Marzo de 2007.

Asimismo, existen otras leyes que desarrollan, inciden y potencian la consecución de esta igualdad. Así tenemos:

- Ley Orgánica del Poder Ejecutivo - Ley $\mathrm{N}^{\circ}$ 29158, publicada el 20 de diciembre de 2007.

- Ley de Organización y Funciones del Ministerio de la Mujer y Poblaciones Vulnerables - Decreto Legislativo $N^{\circ} 1098$, publicada el 20 de enero de 2012.

- Ley Orgánica de Gobiernos Regionales - Ley $\mathrm{N}^{\circ}$ 27867, publicada el 18 de noviembre de 2002 y sus modificatorias.

- Ley Orgánica de Municipalidades - Ley No 27972, publicada el 27 de mayo de 2003.

- Ley General del Sistema Nacional de Presupuesto - Ley $N^{\circ} 28411$, publicada el 08 de diciembre de 2004 y sus modificatorias.

- Ley de Partidos Políticos - Ley No 28094, publicada el 01 de noviembre de 2003.

- Ley de Fomento de la Educación de las Niñas y Adolescentes Rurales - Ley No27558, publicada el 31 de octubre de 2001.

- Ley que fomenta la reinserción escolar por embarazo - Ley $\mathrm{N}^{\circ} 29600$, publicada el 15 de octubre de 2010.

- Texto Único Ordenado de la Ley N 26260 , Ley de Protección contra la Violencia Familiar, publicado el 27 de junio de 1997.

- Ley de Prevención y Sanción del Hostigamiento Sexual- Ley $N^{\circ} 27942$, publicada el 27 de febrero de 2003 y sus modificatorias.

- Ley que modifica el artículo 107 del Código Penal, incorporando el feminicidio - Ley $\mathrm{N}^{\circ}$ 29819, publicada el 27 de diciembre de 2011.

${ }_{13}$ Vicenç Navarro. Bienestar Insuficiente democracia incompleta. Sobre lo que no se habla en nuestro país. Anagrama Barna 2002. 
- Ley contra la Trata de Personas y el Tráfico Ilícito de Migrantes - Ley $\mathrm{N}^{\circ}$ 28950, publicada el 16 de enero de 2007 y su Reglamento el D.S. No 007-2008-IN, publicado el 30 de noviembre de 2008.

- Ley que concede el derecho de licencia por paternidad a los trabajadores de la actividad pública y privada - Ley $\mathrm{N}^{\circ}$ 29409, publicada el 20 de septiembre de 2009.

- Ley de los trabajadores del hogar - Ley $\mathrm{N}^{\circ}$ 27986, publicada el 03 de junio de 2003.

- Ley que incluye el trabajo no remunerado en las cuentas nacionales - Ley $\mathrm{N}^{\circ} 29700$, publicada el 04 de junio de 2011.

- Ley que modifica el artículo 2 de la Ley $\mathrm{N}^{\circ}$ 28457, Leyque regula el proceso de filiación judicial de paternidad extramatrimonial Ley $\mathrm{N}^{\circ} 29715$, publicada el 21 de junio de 2011.

No obstante conviene hacer una mirada hacia las medidas adoptadas hasta que se llega a la aprobación de la ley que marca un hito en las políticas de Igualdad de Oportunidades entre mujeres y hombres en Perú.

Ya en el año 1995 el Perú suscribió los acuerdos de la Plataforma de Beijing concretando así su compromiso con la erradicación de las brechas de género y de toda forma de discriminación por sexo.

A raíz de la suscripción de estos acuerdos, Perú comienza con la incorporación del enfoque de género en las políticas públicas y se crea un Ministerio de la Mujer con el mandato de "diseñar, proponer y ejecutar políticas de desarrollo social y humano promoviendo la equidad de género y la igualdad de oportunidades para la mujer, niñez, adulto mayor y poblaciones en situación de pobreza y pobreza extrema, discriminadas y excluidas".

Mediante ese apoyo a las políticas de Igualdad en Perú se han aprobado diferentes Planes Nacionales de Oportunidades para Mujeres y Hombres. El primer Plan tiene una vigencia entre los años 2000 al 2005, el segundo desde el 2006 al 2010 y el presente cuya vigencia es desde el 2012 al 2017 y que viene determinado por la aprobación de la Ley en el año 2007.
Por tanto el marco normativo actual viene, como hemos dicho, enmarcado en la Ley de Igualdad de Oportunidades que tiene como objetivo "establecer el Marco Normativo, institucional y de políticas públicas en los ámbitos nacional, regional y local, para garantizar a mujeres y hombres el ejercicio de sus derechos a la igualdad, dignidad, libre desarrollo, bienestar y autonomía, impidiendo la discriminación en todas las esferas de su vida pública y privada, propendiendo a la plena igualdad". La ley obliga al Estado Peruano, tanto a nivel nacional, regional y local. (Art. 1)

Hay que destacar que la ley obliga a implementar políticas, programas y acciones encaminadas a conseguir la igualdad entre mujeres y hombres, pero no solo obliga a la implementación, sino también a la evaluación los procesos y los resultados de esa implementación.

El Plan Nacional de Igualdad de Género 2012-2017 (PLANIG) es el instrumento cuyo objetivo es transversalizar el enfoque de género en las políticas públicas del Estado Peruano, en sus tres niveles de gobierno, garantizando la igualdad y la efectiva protección de los derechos humanos para mujeres y hombres, la no discriminación y el pleno desarrollo de las potencialidades y capacidades individuales y colectivas.

El Estado Peruano ha asumido la definición de igualdad de Committee on the Elimination of Discrimination Against Women (CEDAW) y viene clarificando su ordenamiento vigente, tal como se solicita en la recomendación del Comité.

En general, el Estado Peruano ha introducido la definición de CEDAW en documentos oficiales como por ejemplo, la Ley de Igualdad de Oportunidades entre Mujeres y Hombres, precisando que igualdad $y$ equidad son conceptos diferentes. La Ley de Igualdad de Oportunidades se basa en los principios fundamentales de igualdad en su concepción integral, resaltando los derechos igualitarios de las mujeres a lo largo de su ciclo de vida, el respeto a la libertad, dignidad, seguridad y vida humana, y el reconocimiento del 
carácter pluricultural y multilingüe de la nación peruana.

Otra cuestión importante a destacar es la participación en la elaboración del último Plan Nacional de Igualdad 2012-2017 de diversos sectores (17), Gobiernos Regionales (25), organismos públicos (7) y organizaciones de la sociedad civil (19) en su elaboración.

Asimismo, conviene subrayar que todo el marco normativo en Perú se realiza desde la inclusión de la perspectiva de género en sus actuaciones. Así pues, podemos afirmar que las políticas de igualdad se vienen desarrollando sobre los siguientes principios:

"La política de Igualdad de Género alude a la generación de condiciones y medidas establecidas desde el Estado, a fin de garantizar el ejercicio de derechos sociales, económicos, políticos y culturales de todas las personas, teniendo en cuenta el marco del principio universal de igualdad de mujeres y hombres sin distinción ni discriminación".

Las políticas de Igualdad de Género incorporan el enfoque de género, el cual propone desarrollar esfuerzos por transformar a fondo los modelos de políticas públicas predominantes, corregir los desequilibrios existentes, reducir las brechas de género, tomando en cuenta las diferencias entre los géneros y las causas y consecuencias de las desigualdades, legitimadas por las estructuras institucionales y culturales.

Desde esta óptica, la política de Igualdad de Género considera necesario implementar diversas medidas y estrategias tales como ${ }^{14}$ :

\section{POLÍTICAS DE IGUALDAD DE OPORTUNIDADES ENTRE MUJERES Y HOMBRES}

Representan un avance sustantivo frente a las anteriores políticas indiferentes al enfoque de género, buscan eliminar las desigualdades que impiden la participación de las mujeres en la vida política, económica y cultural y garantizar la participación de las mujeres en el ámbito público, con las mismas oportunidades que los hombres (educación, salud, ingresos, etc.)

El objetivo es asegurar igual participación de hombres y mujeres en el proceso de toma de decisiones, igual acceso y control de mujeres y hombres a las oportunidades, recursos y resultados del desarrollo, reducción de la pobreza para hombres y mujeres y mejoramiento del crecimiento económico y desarrollo sostenido en general.

"Medidas positivas o afirmativas que tienen como función favorecer a grupos de mujeres que presentan desventajas $y / o$ privaciones permanentes, mediante mecanismos de eliminación y/o corrección de las discriminaciones y desigualdades reales", como por ejemplo programas de alfabetización dirigidos a las mujeres, mecanismos de cuotas de género en los procesos electorales, incentivos para fortalecer las micro empresas dirigidas por mujeres, etc.

Las medidas positivas o afirmativas permiten eliminar desventajas que afectan a las mujeres, aseguran que todas y todos cuenten efectivamente con los medios, recursos $y$ beneficios específicos de programas sociales $y$ proyectos, y constituyen una estrategia indispensable y complementaria para la igualdad de oportunidades.

\section{LA TRANSVERSALIZACIÓN DEL ENFOQUE DE GÉNERO}

La transversalización del enfoque de género surge con la intención de corregir las políticas de género basadas exclusivamente en la creación de oficinas nacionales y puntos focales, sin involucrar al resto del aparato público, incorporando el enfoque de género en todas las dimensiones de la vida social, política, cultural e institucional.

El objetivo es integrar esta perspectiva desde el diseño, implementación, monitoreo

14 "Conceptos fundamentales sobre el enfoque de género para abordar políticas públicas". Ministerio de la Mujer y Poblaciones Vulnerables. 2012. 
y evaluación de políticas y programas $y$ en todas las esferas y niveles de cualquier acción planificada. Se trata de integrar las consideraciones de género en todos los niveles de la gestión pública, en la planificación, normas y procedimientos, procesos, cultura organizacional, política laboral y presupuesto.

Es considerada en la actualidad, como una de las estrategias de mayor alcance destinada a incluir el enfoque de género en las políticas públicas, debido a su efecto articulador, lo que permite alcanzar mayores resultados $e$ impactos. Incorpora el enfoque de género en todas las dimensiones de la vida social, política, cultural e institucional.

Se desarrolla desde la consolidación de la voluntad política, la institucionalización de mecanismos, la planificación desde la perspectiva de género y el fortalecimiento de capacidades de funcionarios/as, acciones todas que son responsabilidad del Estado y de la sociedad en pleno.

\section{LA INSTITUCIONALIZACIÓN DEL ENFOQUE DE GÉNERO}

La institucionalización del enfoque de género ha adquirido gran importancia ante la necesidad de formalizar los acuerdos y procedimientos en todas las instancias $y$ gestión de las organizaciones. Desde este punto de vista, la institucionalización se encuentra estrechamente asociada a los procesos de transversalización del enfoque de género, considerando el papel que cumplen las instituciones en la interacción humana.
"Institucionalizar el enfoque de género implica el establecimiento de reglas y mecanismos para darle el carácter permanente a una política con enfoque de género, con la intención de convertirla en una práctica regular de la organización. Esto significa que en los procesos de toma de decisiones para la gestión y administración de la organización, se ha incorporado un conjunto de normas $y$ procedimientos que orientan desde

un enfoque de género el proceder de quienes deciden y ejecutan las políticas, planes y programas, transformando la cultura institucional y las concepciones de sus agentes, haciéndolas eficaces y sostenibles."15

Por tanto, según podemos observar, el marco normativo y conceptual en el Perú tiene un desarrollo muy amplio e intenso lo que nos da una idea de la importancia que el tema de la igualdad de oportunidades tiene en estos momentos para la sociedad peruana.

Pero no solamente leyes son las que enmarcan el proceso de avance de la igualdad en Perú, existe un Ministerio de la Mujer que tiene como principal objetivo promover la equidad de género y la igualdad de oportunidades para la mujer.

La Ley de Igualdad de oportunidades vigente tiene como objetivo, "establecer el Marco Normativo institucional de políticas públicas en los ámbitos nacional, regional y local, para garantizar a mujeres y hombres el ejercicio de sus derechos a la igualdad..." La Ley contempla la necesidad de incorporar a los hombres en la conquista de la igualdad de oportunidades de las mujeres, y ello no puede dejar de ser el mejor signo de avance en el trabajo para la igualdad efectiva. Pero según nuestra experiencia hay que trabajar en dos líneas muy claras y diferenciadas que deben ser la base de las políticas de igualdad de oportunidades en la sociedad peruana.

1. La formación de las mujeres y de los hombres para llegar a comprender lo que significa la igualdad efectiva entre los sexos, y la necesidad de tener consciencia de que existe una desigualdad que hay que corregir.

2. La creación de herramientas de ayuden a la sociedad en general y a las empresas, a los centros educativos, a las universidades a desarrollar acciones encaminadas a determinar el nivel de desigualdad y a la vez la manera en que se puede corregir.

\footnotetext{
15 Plan Nacional de Igualdad de género 2012-2017. Agosto 2012.
} 
Las políticas de igualdad de oportunidades entre mujeres $y$ hombres representan un avance sustantivo frente a las políticas que no tienen ni tenían el enfoque de género, ni tampoco preveían la participación de la mujer en la vida política, económica y cultural. El objetivo de las políticas de igualdad es asegurar la participación de mujeres y hombres en el proceso de toma de decisiones, y en la mejora del crecimiento económico y desarrollo sostenible.

No obstante, también se debe tener en cuenta que para que la igualdad de oportunidades tenga un desarrollo pleno es necesario dotar de recursos para su implementación y la limitada asignación de recursos humanos especializados y la escasa inversión pública siguen siendo aspectos centrales que evidencian las debilidades en su institucionalización.

Por eso debemos dotarnos de herramientas que permitan avanzar hacia la plena igualdad de derechos en todos los ámbitos y especialmente en el laboral.

\section{MARCO COMPARATIVO CON EUROPA Y LATINOAMÉRICA}

Existen diversos países tanto en Europa como en Latinoamérica donde se ha regulado la igualdad de oportunidades entre hombres y mujeres. Conviene destacar que países con un fuerte desarrollo económico no tienen Ley de Igualdad, tanto en Europa como en Latinoamérica. (ejemplo Chile, Francia, Alemania). Asimismo, también conviene plantear que las leyes de Igualdad en los diferentes países suelen tener un tronco común, pero en los contenidos hay diferencias.

Algunas cuestiones a destacar son:

Finlandia exige tener un plan de igualdad en los centros educativos.

España tiene en su ley la obligatoriedad de los planes de igualdad para empresas de más de 250 trabajadores y recomienda y potencia para los de menos de 250
Suecia uno de los países más igualitarios tiene ley igualdad desde 1991. Entre las medidas relacionadas con la igualdad salarial, la Ley impone al empleador la obligación de controlar y analizar anualmente las disposiciones y las prácticas relativas al salario, y las diferencias salariales entre hombres y mujeres que realizan trabajo de igual valor

Suecia tiene uno de los más altos niveles del mundo en igualdad entre los sexos. Esta visión se funda en la convicción de que cuando mujeres y hombres comparten igualmente el poder y la influencia, se abre el camino de una sociedad más justa y democrática. Un sistema de bienestar desarrollado facilita a ambos sexos el equilibrio entre su trabajo y su vida familiar.

Existen leyes de Igualdad en el área Latam en Bolivia, Perú, Ecuador, siendo la de Perú una de las más completas y desarrolladas.

En la ley española es donde aparecen los planes de igualdad para la empresas y es una experiencia positiva que sería exportable a otros países como una forma de ir ahondando en la igualdad laboral con una herramienta estratégica dentro de los planes estratégicos generales de las empresas.

\section{HERRAMIENTAS DE IGUALDAD. UNA HERRAMIENTA EN ESPAÑA LOS PLANES DE IGUALDAD EN LA EMPRESA}

La Ley Orgánica 3/2007, de 22 de marzo, para la igualdad efectiva entre mujeres y hombres (en adelante, LOI) se hace eco de la necesidad patente de que en el avance en la igualdad se involucren las empresas. Por ello regula los Planes de Igualdad en las empresas y su negociación en los convenios colectivos.

Los planes de igualdad tienen como objetivo principal establecer criterios básicos para la aplicación de la igualdad de oportunidades en el ámbito laboral desde una perspectiva de género y de forma transversal para involucrar a todo el personal de la empresa. Para desarrollar un plan de igualdad en una empresa es fundamental implicar a todo el personal de recursos humanos, los agentes 
sociales y todas las personas con poder de decisión.

Los planes de igualdad promueven acciones dentro de la organización de la empresa que deben propiciar un cambio en el modo de pensar y de actuar. Visualizar la posible discriminación existente es el primer paso para empezar a trabajar en sensibilización. Este trabajo se lleva a cabo con el desarrollo de un diagnostico que debe medir diferentes ámbitos de la empresa: la selección de personal, la descripción de los lugares de trabajo, la formación en la propia empresa, los niveles salariales en las distintas categorías, la promoción, la salud en el trabajo,... El diagnóstico debe medir la necesaria situación de mujeres y hombres en el lugar de trabajo y las posibles diferencias existentes por razón de sexo y género.

Después de haber analizado los datos obtenidos y sus correlaciones entre las mujeres y los hombres, se lleva a cabo el plan de acción que implica el establecimiento de objetivos a corto, medio y largo plazo y se diseñan las acciones para llevar a cabo.

Para llevar a cabo un plan de igualdad tiene que haber la voluntad clara y decidida de la dirección de la empresa y asignación de recursos humanos y materiales. Los contenidos que podría tener un plan de igualdad de oportunidades serían:

- Formación del personal.

- Incorporación del uso no sexista del lenguaje.

- Revisión de la imagen corporativista de la empresa.

- Desagregación por sexos de la información.

- Procesos de selección que fomenten la igualdad de oportunidades.

- Promoción de la carrera profesional de las mujeres.

- Medidas de apoyo a la conciliación de la vida familiar- personal y laboral.

- Prevención del acoso moral y sexual en el trabajo.

- Comunicación interna y difusión sobre temas relacionados con la igualdad de oportunidades.
- Creación de equipos de trabajo o comisiones que impulsen la igualdad de oportunidades en la organización.

- Participación de la organización en proyectos desarrollados por la administración pública en igualdad de oportunidades.

- Acciones de formación específica e incorporación transversal de igualdad de oportunidades en la política de recursos humanos de la organización.

- Elaboración de manuales que especifiquen la transversalidad de las acciones de igualdad de oportunidades.

Para que estas $\mathrm{u}$ otras acciones sean efectivas, las cúpulas directivas y el personal de las empresas necesitan estar sensibilizadas, tener conocimientos y herramientas para incorporar la igualdad de oportunidades entre mujeres y hombres como un elemento de calidad, de mejora, de eficacia, de eficiencia y rentabilidad.

Por lo tanto, sería conveniente que del mismo modo que existen procesos de calidad y procesos de gestión medioambiental integrados en la gestión de la empresa, se empiece a incorporar un sistema de gestión para la igualdad de oportunidades. Solamente de esta manera las empresas serán realmente acogedoras para las mujeres, pero no nos olvidemos, también para los hombres.

\section{ALGUNAS BUENAS PRÁCTICAS}

"La igualdad mejora el ambiente de trabajo y las relaciones laborales. Y se reduce el ausentismo laboral", afirma la directora general Navarro. También pueden servir para dar brillo a la cuenta de resultados. (Esa es la experiencia de Autoescuela Placentina, una empresa familiar con cinco autoescuelas y un centro de formación de conductores de vehículos pesados y mercancías peligrosas en la provincia de Cáceres (España). Para potenciar la llegada de clientas en el año 2000 ofrecieron servicio de guardería durante las clases. Y llegaron las alumnas. "Si les dábamos facilidades, las mujeres se matriculaban", relata María del Puerto Macedo, una de las responsables del negocio. Las alumnas, que antes eran el $20 \%$ de los matriculados, ya son el 60\%, según Macedo. 
La empresa extendió el servicio de guardería gratuito a sus trabajadores -22 hombres y ocho mujeres-. También optó por horarios flexibles y adecuados a la demanda. El resultado es que "no hay malas caras". "El ambiente de trabajo ha mejorado, se rinde más. Existe más motivación y menos absentismo. La igualdad cuesta dinero al principio, pero luego es rentable", resume Macedo.

"Calidad e igualdad forman parte de la excelencia empresarial", defiende Dolors Salguero, jefa de recursos humanos del Grupo Borges (más de 700 empleados). Planteó los planes de equiparación "antes de que se aprobara la ley Śde Igualdad, vigente desde 2007Ș". Ofrecen cursos de formación para carretilleras, elección de horarios para trabajadores de jornada reducida, una hora para comer -"así la gente se va antes a casa"y horas libres para llevar al niño al médico, por ejemplo.

Para facilitar la presencia femenina en el escalón más alto -algo que, para Salguero, difícilmente se puede imponer con cuotas- han dado un paso más: la formación de futuras directivas.

\section{CONCLUSIONES}

No quisiéramos acabar este artículo sin centrar nuestros argumentos en las conclusiones siguientes:

1. Perú es un país que está alcanzando un nivel importante de desarrollo democrático lo que lo posiciona en una situación de mejora de todos los derechos civiles y sociales.

2. Consciente de este avance, Perú tiene un historial que demuestra su alto nivel de compromiso con la lucha por la igualdad de oportunidades entre mujeres y hombres. Pero en este proceso la sociedad civil, los grupos de mujeres también tienen su importante papel de recordar la necesidad de alcanzar los niveles adecuados de igualdad en el desarrollo social.

3. Las estadísticas, los datos, las leyes, el diagnóstico está ya elaborado en Perú. En este artículo solamente hemos hablado de los aspectos laborales, pero se trabaja también en otros ámbitos como son los de la violencia, los de la salud, la educación.

4. La comparación de Perú con otros países de Latinoamérica es muy interesante porque destaca la gran proliferación de países que ya han actuado y que han empezado a legislar en los aspectos de igualdad de oportunidades

5. El proceso no tiene vuelta atrás, pero ahora se hace imprescindible profundizar en las políticas, asegurar que se lleven a cabo, potenciar la evaluación de los indicadores, la formación, el desarrollo de herramientas.

6. En Europa especialmente, sobre todo en los países nórdicos, se han llevado a cabo modelos de potenciación de la igualdad intervencionistas porque era evidente que el avance espontáneo de la igualdad en la sociedad no era, ni mucho menos, rápido. En estos países se han llevado a cabo políticas tendentes a que en la sociedad se precipite la igualdad de oportunidades. En los EEUU las políticas fueron mucho más rotundas con las acciones de discriminación positiva que se llevaron a cabo sobre todo en los ámbitos laborales y educativos.

7. Ahora le toca a los países de Latinoamérica, Perú uno de ellos. Estos países tienen grandes oportunidades para llevar a cabo acciones que en un día quizás no tan lejano alcancen la igualdad entre mujeres y hombres. Les pueden servir los errores pero también los aciertos de otras experiencias europeas o anglosajonas. Hay que rescatar lo bueno y procurar no caer en lo malo hecho.

8. En nuestro artículo proponemos los planes de acción de igualdad que estamos convencidas son útiles, y lo son porque siguen un proceso lógico de análisis. Primero saber qué ocurre, evaluar las posibles situaciones anómalas y desiguales. Después elaborar un plan de acción basado en estos análisis. Pero lo más importante de todo, es que los planes de igualdad deben ser realistas, deben ayudar a las empresas, 
deben ajustarse a la cultura existente, no pueden ser intrusos y sobretodo no pueden ser una herramienta que cree actitudes en contra de las mujeres, los planes de igualdad deben ser una herramienta aliada para la igualdad y para ello la clave es incorporar a los hombres en todos los procesos.

\section{REFERENCIAS}

Diario El País: La Igualdad atrae clientes17/04/11. Madrid. España on line.

Morondo Taramundi, Dolores Derecho comparado: Bélgica, Canadá y Suecia. Emakunde. Eusko Jaurlaritza/Gobierno Vasco. Con La cofinanciación del Fondo Social Europeo

Emakume Eta GizoneN BerdintaSunerako Aholkularitzan Prestakuntza Eta KReditazioa Formación y Acreditación en Consultoría Para La Igualdad de Mujeres Y Hombres País Vasco (España)

CEPAL, (2008) Estadísticas de América Latina y El Caribe. Naciones Unidas. Nueva York, EE. UU.

Fundación INCYDE (2010) Igualdad de Oportunidades. Madrid. España.

INEI. Estadísticas con enfoque de género. Lima. Perú. 2011. www.inei.gob.pe/web/Biblioinei/ Boletines

Instituto de la Mujer (2008) Ministerio de Trabajo y Asuntos Sociales. "Manual para elaborar un Plan de Igualdad en la empresa. Aspectos básicos". Madrid. España.

Instituto de la Mujer (2007) Orientaciones para Negociar Medidas y Planes de Igualdad de Oportunidades entre mujeres y hombres en las empresas. Madrid. España.

Ley de Igualdad de Oportunidades (2007) Madrid, España.

Ley de Igualdad de Oportunidades (2007) Lima, Perú.

MIMDES (2010) Brechas de género en el uso del tiempo. Freyre, M y López, E., Lima, Perú

MIMDES-INEI. I (2010) Encuesta Nacional de Uso del Tiempo., Lima, Perú

Ministerio de la Mujer y Poblaciones Vulnerables (2012) Conceptos fundamentales sobre el enfoque de género para abordar políticas públicas". Lima. Perú

Ministerio de Vivienda, Construcción y Saneamiento. Informe DS 027- 2007-PCM. Enero 2012. Lima, Perú

Plan Nacional de Igualdad de Género 2012-2017. Agosto 2012 Lima, Perú

SWEDEN. SE: INSTITUTO SUECO www.sweden.se

SECRETARÍA GENERAL DEL GOBIERNO SUECOSWEDISH TRADE COUNCILINVEST 2013 Estocolmo. Suecia

Vicenç Navarro (2002) Bienestar Insuficiente democracia incompleta. Sobre lo que no se habla en nuestro país. Anagrama Barna. Barcelona. España www.weforum.org. 